\title{
Rebitagem por Fricção ("FricRiveting") de Liga de Alumínio 6056 T6 e Poliamida 6: Influência da Velocidade de Rotação na Formação da Zona de Ancoragem e no Desempenho Mecânico ${ }^{a}$
}

Bruno Cordeiro de Proença ${ }^{1}$, Lucian Blaga² Jorge Fernandez dos Santos², Leonardo Bresciani Canto', Sergio de Traglia Amancio Filho ${ }^{2,3}$

1 Programa de Pós-graduação em Ciência e Engenharia de Materiais, Universidade Federal de São Carlos - UFSCar, São Carlos, SP, Brasil.

2 Centre for Materials and Coastal Research, Institute of Materials Research, Materials Mechanics, Solid State Joining Processes, Helmholtz-Zentrum Geesthacht, Geesthacht, Germany.

3 Institute of Polymer Composites, Hamburg University of Technology, Hamburg, Germany.

Recebido: 03 Nov., 2015

Aceito: 15 Dez., 2015

E-mails: brunocoproenca@gmail.com (BCP), leonardo@ufscar.br (LBC), sergio.amancio@hzg.de (STAF) a Contribuição do CONSOLDA 2014.

Este é um artigo publicado em acesso aberto (Open Access) sob a licença Creative Commons Attribution Non-Commercia, que permite uso, distribuição e reproduçãa em qualquer meio, sem restriçōes desde que sem fins comerciais e que o trabalho original seja corretamente citado.
Resumo: O uso de estruturas híbridas metal-polímero é uma alternativa para a redução de peso e de consumo de combustível na indústria de transporte que visa minimizar a emissão de gases nocivos ao efeito estufa. A Rebitagem por Fricção ('Friction Riveting) é uma técnica relativamente nova para união de estruturas híbridas metal-polímero. O processo baseia-se na geração de calor friccional entre os componentes resultando na deformação plástica da extremidade do rebite metálico, que é ancorado dentro do componente polimérico. O presente estudo avaliou a viabilidade técnica da união de AA 6056 T6 e PA6, com foco na influência da velocidade de rotação do rebite no desempenho mecânico das juntas. A máxima temperatura atingida no processo aumentou com o acréscimo na velocidade de rotação, de $291 \pm 6^{\circ} \mathrm{C}$ com $10000 \mathrm{rev} / \mathrm{min}$ para $375 \pm 5^{\circ} \mathrm{C}$ com $15000 \mathrm{rev} / \mathrm{min}$. A utilização de maiores valores de velocidade de rotação causou a deformação plástica da ponta do rebite durante a fase de fricção. Isso levou a juntas mecanicamente mais resistentes, devido à maior ancoragem do rebite metálico na placa polimérica. As juntas de AA 6056 T6-PA6 apresentaram bom desempenho de resistência à tração atingindo 85\% da resistência à tração do rebite metálico. Portanto, verificou-se que é possível unir AA 6056 T6 e PA6 pela técnica de rebitagem por friç̧ão e que a velocidade de rotação influencia diretamente a resistência à tração das juntas.

Palavras-chave: Estruturas híbridas; Rebitagem por fricção; AA 6056 T6; Poliamida 6.

\section{Friction Riveting of Aluminum Alloy $6056 \mathrm{~T} 6$ and Polyamide 6: Role of the Rotation Speed on the Formation of the Anchoring Zone and Mechanical Performance}

\begin{abstract}
Hybrid metal-polymer structures are an alternative solution to reduce weight and fuel consumption in the transportation industry in order to minimize the emission of noxious gases in regard to the greenhouse effect. Friction Riveting is a relatively new technique for joining metal-polymer hybrid structures. The process relies on the generation of frictional heat between the components causing the plastic deformation of the metallic rivet and its anchoring in the polymer component. This study evaluated the technical feasibility of friction-riveted AA 6056 T6 and PA6 joints, and the influence of the rotational speed (RS) on the maximum process temperature and on the mechanical performance of the joints. The maximum temperature reached increased with the rotational speed, from $291 \pm 6{ }^{\circ} \mathrm{C}$ at $10000 \mathrm{rev} / \mathrm{min}$ to $375 \pm 5^{\circ} \mathrm{C}$ at $15000 \mathrm{rev} / \mathrm{min}$. The use of greater rotational speeds induced the plastic deformation of the tip of the metallic rivet during the frictional phase. This led to mechanically stronger joints due to the larger anchoring of the metallic rivet within the polymeric plate. The AA 6056 T6-PA6 joints had good tensile strength, achieving $85 \%$ of the metallic rivet's tensile strength. Therefore, the feasibility of friction-riveted AA 6056 T6-PA6 joints was proven. Furthermore, it was shown that the rotational speed influences directly the rivet anchoring and thus the tensile strength of the joints.
\end{abstract}

Key-words: Hybrid structure; Friction riveting; AA 6056 T6; Polyamide 6. 


\section{Introdução}

A indústria de transportes busca reduzir o peso dos veículos visando menor consumo de combustível e, consequentemente, reduzir a emissão de gases nocivos do efeito estufa. O desenvolvimento de termoplásticos de engenharia para uso na indústria de transportes resultou na substituição parcial de diversas peças metálicas por poliméricas. Atualmente, cerca de $16 \%$ do peso de um veículo consiste em materiais poliméricos, com tendência de crescimento para $25 \%$ nos próximos 5 anos [1]. Técnicas de união para estruturas híbridas metal-polímero são essenciais para a incorporação de peças poliméricas no setor automotivo [2,3].

Atualmente, os processos de união híbrida metal-polímero utilizados industrialmente são a fixação mecânica tradicional, a colagem por adesivos ou uma combinação de ambos, além do processo de sobreinjeção. A fixação mecânica tradicional é um processo mais rápido e barato que a união por adesivos. Os componentes, no caso da colagem, precisam passar por tratamentos superficiais como jateamento de areia ou decapagem ácida para melhorar a adesão entre os componentes [4-6]. Por outro lado, as juntas coladas apresentam menor peso quando comparadas com os métodos tradicionais de conexão mecânica [5]. Apesar das vantagens, essas técnicas apresentam algumas limitações. No caso do uso de adesivos, altos custos, longos tempos de cura da resina resultam em ciclos longos de união, além de baixa previsibilidade da durabilidade de juntas coladas por adesivos [5-7]. Já no caso da fixação mecânica, há concentração de tensão devido à presença do furo passante ao redor do elemento de fixação, o que pode gerar falha prematura, além do processo consistir de várias etapas [4,8,9].

Nas últimas décadas, novas técnicas para a união de componentes metálicos com componentes poliméricos foram desenvolvidas. Técnicas baseadas na geração de calor por fricção têm chamado a atenção devido aos menores gastos energéticos destas quando comparadas com as demais. $\mathrm{O}$ instituto alemão Helmholtz-Zentrum Geesthacht (HZG) tem se dedicado a desenvolver e aplicar novas técnicas para a união híbrida metal-polímero que visam sanar restrições das técnicas convencionais de fixação mecânica e colagem por adesivos. A Rebitagem por Fricção ('Friction Riveting, FricRiveting') [10], União Pontual por Fricção ('Friction Spot Joining- FSpJ') [11], Rebitagem por Injeção baseada em Energia Friccional ('Friction-based Injection Clinching Joining, F-ICJ') [12] e Rebitagem Plástica por Friç̧ão ('Friction Staking') [13] tiveram suas viabilidades técnicas comprovadas para uma série de combinações de ligas metálicas leves com materiais poliméricos e compósitos poliméricos de interesse da indústria de transportes.

A rebitagem por friç̧ão (do Inglês 'Friction Riveting') é uma técnica inovadora para união híbrida metal-polímero. Esta foi desenvolvida e patenteada em 2007 por Amancio et al. [14] pelo instituto alemão Helmholtz-Zentrum Geesthacht (HZG). A rebitagem por fricção combina os princípios da fixação mecânica com os das técnicas de soldagem por friç̧ão. A Figura 1 apresenta um esquema simplificado das etapas da rebitagem por fricção para uma junta de inserto metálico em placa de polímero termoplástico. O rebite metálico é rotacionado e pressionado contra a placa polimérica termoplástica. (Figura 1b). Durante esta fase, calor é gerado por fricção aumentando localmente a temperatura na ponta do rebite. Forma-se uma fina camada de polímero amolecido (se o polímero

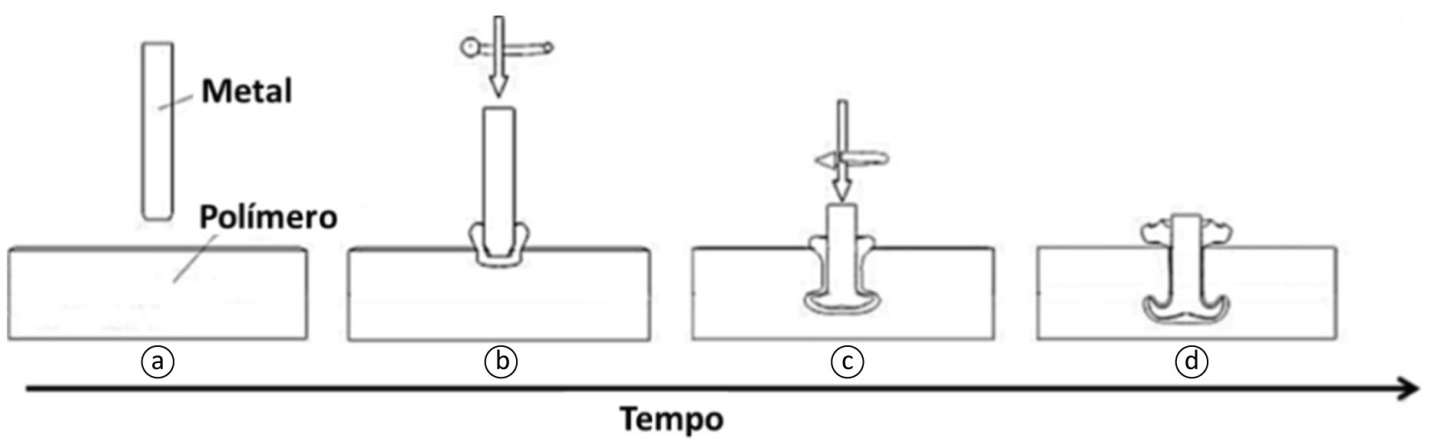

Figura 1. Esquema simplificado da técnica de rebitagem por fricção para do tipo inserto metálico em placa polimérica. Posicionamento inicial dos componentes (a). Rebite metálico rotativo é inserido na placa polimérica expelindo o polímero amolecido (b). Desaceleração da rotação e aplicação de força axial para se deformar a ponta do rebite plasticamente e formar a zona de ancoramento (c). Consolidação da junta após resfriamento sob pressão (d). Adaptado de [15]. 
Rebitagem por Friç̧ão ("FricRiveting”) de Liga de Alumínio 6056 T6 e Poliamida 6: Influência da Velocidade de Rotação na Formação da Zona de Ancoragem e no Desempenho Mecânico

for amorfo) e/ou fundido (se o polímero for semicristalino) ao redor do rebite metálico. Com a aplicação contínua da força axial e rotação, o rebite metálico penetra a placa polimérica, expelindo o polímero amolecido/fundido da placa em forma de rebarba. A temperatura na ponta do rebite aumenta progressivamente pois a taxa de geração de calor se torna muito maior do que a taxa de dissipação do mesmo. Isso ocorre devido à baixa condutividade térmica dos polímeros. Ao final da fase de fricção, a temperatura na ponta do rebite é suficiente para permitir sua deformação plástica sob uma força axial. Nesse ponto, a velocidade de rotação do rebite é desacelerada para zero e a força axial é aumentada para um valor definido (nesse caso chamada força de forjamento) ou mantido igual à força da fase de friç̧ão (Figura 1c). A ponta do rebite se deforma plasticamente assumindo uma geometria paraboloidal, com diâmetro maior do que o original, formando, assim, a zona de ancoragem. Após isso, a junta é consolidada sob pressão (Figura 1d). Os principais mecanismos de união deste processo são: ancoragem mecânica e forças adesivas na interface metal-polímero [10,14,16].

A viabilidade técnica da rebitagem por fricção foi comprovada para diversas combinações de materiais, dentre elas, poli(éter imida) (PEI) e AA 2024 T351 [10], policarbonato (PC) e AA 2024 T351 [17], compósito de poli(éter imida) reforçada com fibra de vidro (PEI-FV) e titânio grau 2 [15], compósito poli(éter-éter-cetona) PEEK reforçado com fibra curta de carbono e titânio grau 3 [18], e compósito pultrudado de poliéster termofixo reforçado com fibra de vidro e liga Ti6Al4V [19]. Essas juntas foram fabricadas em um equipamento que consiste em um cabeçote de soldagem controlado por tempo. Os três parâmetros principais são: velocidade de rotação (VR), tempo de união (TU) e pressão de união (PU). A velocidade de rotação é a velocidade angular do rebite, e influencia na geração de calor, que por sua vez tem efeito sobre a plastificação do rebite, a viscosidade do polímero e a extensão de sua degradação. A pressão de união (PU) é a soma da pressão de fricção (PF) e a pressão de forjamento (PFo). $O$ tempo de união consiste na soma dos tempos de fricção (TF) e de forjamento (TFo). O tempo de fricção (TF) é o período no qual o rebite rotaciona penetrando a placa polimérica a uma pressão constante (PF). Esta é a etapa de maior influência na geração de calor e também de maior influência na geração de defeitos volumétricos relacionados à termodegradação do polímero. O tempo de forjamento (TFo) é o período de aplicação da pressão de forjamento (PFo). Esta etapa possui grande influência na formação de defeitos na interface metal-polímero devido à contração diferencial entre o metal e o polímero durante a consolidação [10,18].

O presente trabalho investigou a viabilidade técnica da união de rebite de liga de alumínio 6056 T6 e chapa de poliamida 6 (PA6) pelo processo de rebitagem por fricção com foco nos efeitos da velocidade de rotação do rebite (VR) na geração de calor e na resistência à tração das juntas. Poliamida 6 é um termoplástico de engenharia que vem sendo amplamente utilizado na indústria automobilística devido ao seu bom desempenho mecânico [20]. A liga de alumínio 6056-T6 é muito utilizada na indústria automobilística na forma de fixadores, como rebites e parafusos, devido à elevada resistência específica e resistência à corrosão [21]. Essas juntas foram produzidas em um novo equipamento de rebitagem por fricção no instituto alemão Helmholtz-Zentrum Geesthacht (HZG). Tal equipamento tem como vantagem permitir a execução do processo de rebitagem por fricção através do controle por tempo, força e deslocamento do mandril, ou por uma combinação desses parâmetros [16]. Os parâmetros de processo utilizados neste novo equipamento são [16]: deslocamento na friç̧ão (DaF), força de fricção (FF) e força de forjamento (FFo). O deslocamento na fricção (DaF) é a distância percorrida pelo mandril durante a fase de fricção. Isso implica que a desaceleração da velocidade de rotação (VR) ocorrerá quando o mandril atingir o deslocamento axial desejado. Esse parâmetro não pode ser relacionado diretamente com o tempo de friç̧ão (TF) usado na variante controle por tempo, pois o uso de diferentes forças de friç̧ão (FF) e velocidades de rotação (VR) fazem a duração da fase de fricção variar. Sendo assim, ao se adotar o DaF, o processo passa a ser controlado pela força e limitado pela posição do rebite (force-controlled process limited by position) e não mais pelo tempo (time-controlled process). As forças de fricção (FF) e forjamento (FFo) podem ser diretamente relacionadas com as pressões de fricção (PF) e de forjamento (PFo), respetivamente, pois esses parâmetros representam a carga axial aplicada durante o processo de união.

\section{Materiais e Métodos}

\subsection{Materiais}

Foram utilizados pré-formas industriais de rebites extrudados de alumínio 6056 T6 como rebite, e placa extrudada de poliamida 6. Os materiais foram fornecidos pela empresa EJOT GmbH, Alemanha, por meio de um projeto de cooperação industrial. A liga de alumínio 6056 faz parte da família de ligas Al-Si-Mg (AA 6XXX). O uso 
de magnésio e silício torna a liga AA 6056 tratável termicamente, aumentando a sua resistência mecânica [22]. A poliamida 6 é um termoplástico semicristalino com ampla aplicação na indústria de transporte [23]. A poliamida 6 possui bom balanço de propriedades, como rigidez, dureza, resistência química, ao desgaste e estabilidade térmica, já região amorfa contribui para a resistência ao impacto e à elongação tendo a temperatura de transição vitrea em torno de $50^{\circ} \mathrm{C}$ e ponto de fusão em torno de $220^{\circ} \mathrm{C}$ [24].

A Figura 2 apresenta as geometrias e dimensões do rebite de AA 6056 T6 (Figura 2a) e da placa de PA6 (Figura 2b) utilizadas.

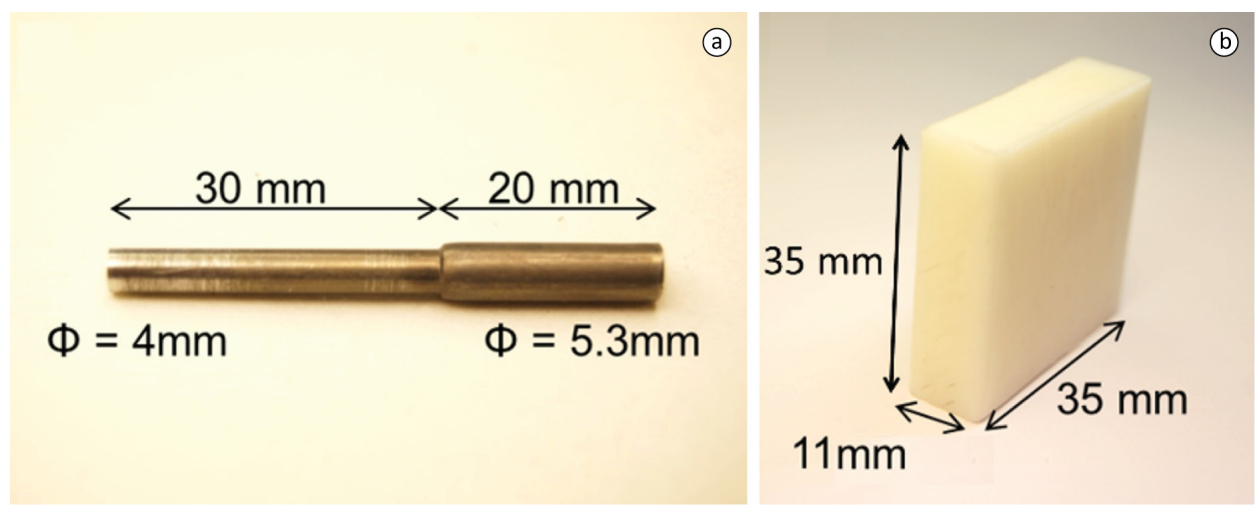

Figura 2. Rebite metálico de AA 6056 T6 (a) e Placa de poliamida 6 (b).

\subsection{Equipamento e procedimento de união}

As juntas rebitadas por fricção de AA6056 T6 e PA6 foram produzidas no novo maquinário (RSM 410, Harms \& Wende $\mathrm{GmbH}$, Alemanha). Tal equipamento consiste no cabeçote de soldagem modelo RSM 410 (Harms e Wende GmbH Co. KG. Hamburg/Alemanha) montado em uma estrutura do tipo portal automatizada. Esse equipamento possui sensor de força tri-axial, sensores de posição integrados e uma mesa móvel nos eixos $X$ e $Y$ com espaço de trabalho de aproximadamente $1,5 \mathrm{~m}^{2}$. Esta automação do processo torna-o mais adequado para aplicações que exijam flexibilidade do equipamento, unindo juntas desde escala laboratorial até grandes componentes. Além disso, o novo equipamento permite a divisão do processo em cinco etapas controladas (as três primeiras estão relacionadas com a fricção, a quarta é uma fase intermédia e a quinta está relacionada com o forjamento), enquanto na configuração anterior, a união ocorria em duas únicas etapas (fricção e forjamento).

Três condições de processo foram utilizadas, como apresentado na Tabela 1. Tais condições foram selecionadas baseando-se em teste preliminares para esta combinação de material. Este estudo visa analisar a influência da velocidade de rotação (VR) nas juntas rebitadas por fricção, logo, este parâmetro foi o único alterado. Três velocidades de rotação foram selecionadas: mínima de $10000 \mathrm{rev} / \mathrm{min}$, média de $12500 \mathrm{rev} / \mathrm{min}$ e máxima de $15000 \mathrm{rev} / \mathrm{min}$. Uma termo-câmera de radiação infravermelho (modelo High-end Camera Series ImageIR fabricada pela Infratech $\mathrm{GmbH}$, Alemanha) foi utilizada para monitorar a evolução da temperatura durante o processo de rebitagem por friç̧ão. A temperatura foi medida no material polimérico expelido em forma de rebarba da região da união. Utilizou-se um filtro de temperaturas entre $150^{\circ} \mathrm{C}$ e $700^{\circ} \mathrm{C}$ e aquisição de dados à $60 \mathrm{~Hz}$. As medições foram realizadas na mesma posição para todas as condições. Três replicas foram produzidas para cada condição estudada.

Tabela 1. Condições de rebitagem por friç̧ão empregadas para as juntas de AA 6056-T6 e PA6.

\begin{tabular}{|c|c|c|c|c|c|}
\hline & $\begin{array}{c}\text { Velocidade de } \\
\text { Rotação - VR } \\
\text { [rev/min] }\end{array}$ & $\begin{array}{c}\text { Tempo de } \\
\text { Fricção-TF [ms] }\end{array}$ & $\begin{array}{c}\text { Tempo de } \\
\text { Forjamento - TFo } \\
\text { [ms] }\end{array}$ & $\begin{array}{l}\text { Força de Fricção - FF } \\
\text { [N] }\end{array}$ & $\begin{array}{c}\text { Força de Forjamento } \\
- \text { FFo }[N]\end{array}$ \\
\hline C1 & 10000 & & & & \\
\hline$C 2$ & 12500 & 3500 & 3000 & 1500 & 2000 \\
\hline $\mathrm{C} 3$ & 15000 & & & & \\
\hline
\end{tabular}


Rebitagem por Fricção ("FricRiveting") de Liga de Alumínio 6056 T6 e Poliamida 6: Influência da Velocidade de Rotação na Formação da Zona de Ancoragem e no Desempenho Mecânico

\subsection{Caracterização microestrutural}

Análises geométricas da zona de ancoragem do rebite foram realizadas com a utilização de estereomicroscópio (Leica DC 300). Inicialmente, o rebite metálico foi cortado rente à placa polimérica para eliminar o excesso de material visando uma fácil manipulação das amostras. Em seguida, a junta foi seccionada ao meio utilizando um disco de corte de diamante de baixa velocidade (ISOMET, Buehler GmbH, Alemanha). As amostras foram preparadas seguindo procedimento metalográfico tradicional (embutimento, lixamento e polimento). Com as imagens, as medidas de penetração e deformação do rebite metálico na placa polimérica foram quantificadas utilizando o software Image J. Também foram quantificadas as medidas necessárias para o cálculo da eficiência de ancoragem utilizando-se o modelo de razão volumétrica de ancoragem (Figura 3) proposto por Blaga et al. [15].

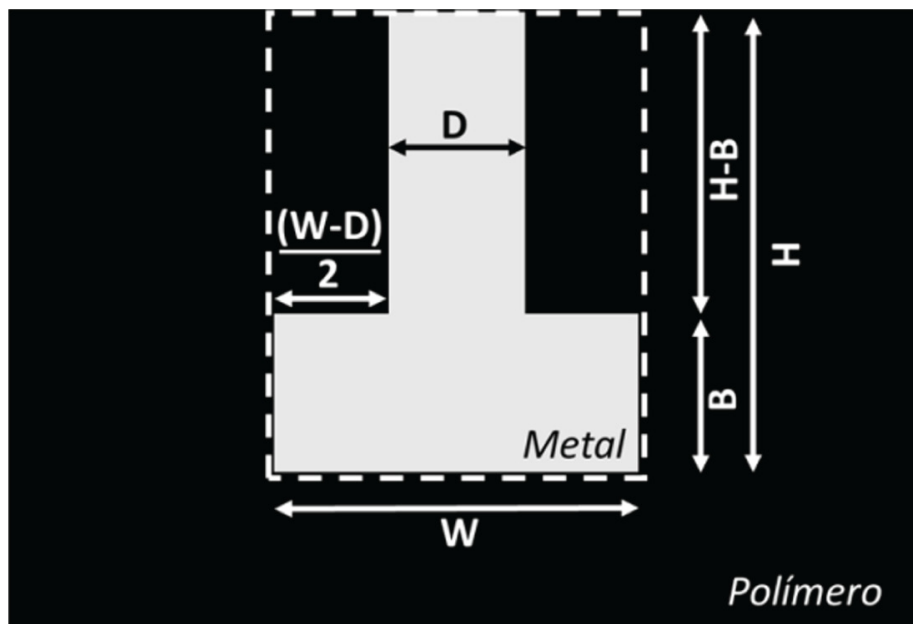

Figura 3. Detalhamento da região de união segundo o modelo geométrico de razão volumétrica de ancoragem para juntas do tipo inserto metálico em polímero rebitadas por fricção Adaptado de [11].

A razão volumétrica de ancoragem (RV) considera o volume do polímero que está acima da zona deformada da ponta do rebite e é calculada utilizando a Equação 1.

$$
\mathrm{RV}=\frac{(\mathrm{H}-\mathrm{B}) *(\mathrm{~W}-\mathrm{D})}{\mathrm{W}^{2} * \mathrm{H}}
$$

Na Equação 1, H é a profundidade de penetração do rebite metálico na placa polimérica, B é a altura da ponta deformada do rebite, W é a largura de deformação da ponta do rebite e D é o diâmetro inicial do rebite.

\subsection{Caracterização mecânica}

A resistência mecânica das juntas foi determinada por ensaio de tração ('T-pull') baseado na norma ISO 6892-84 [25] e proposto por Amancio-Filho [26]. Foi utilizado uma máquina universal de ensaios Zwick 1478 (Zwick Roell AG, Alemanha) com velocidade da travessa de $1 \mathrm{~mm} / \mathrm{min}$, a temperatura ambiente $\left(21^{\circ} \mathrm{C}\right)$. A geometria da junta ensaiada (junta do tipo inserto metálico) é ilustrada na Figura 4a e o porta-amostra usado para o ensaio de tração é representado na Figura 4b, a distância entre os fixadores é de $30 \mathrm{~mm}$.

\section{Resultados e Discussão}

\subsection{Influência da velocidade de rotação na temperatura processual}

A Figura 5 apresenta um exemplo da medição da temperatura usando uma termo-câmera de radiação infravermelho para a condição C2. 


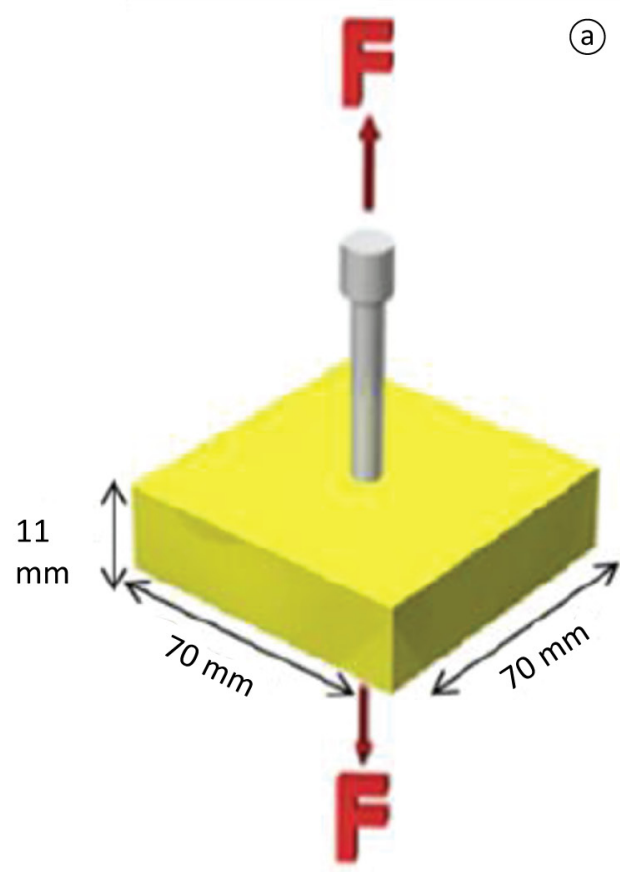

(a)

Figura 4. Amostra para ensaio de tração (T-pull) (a) e porta amostra utlizado no ensaio de tração (b). Adaptado de [27].

(a)
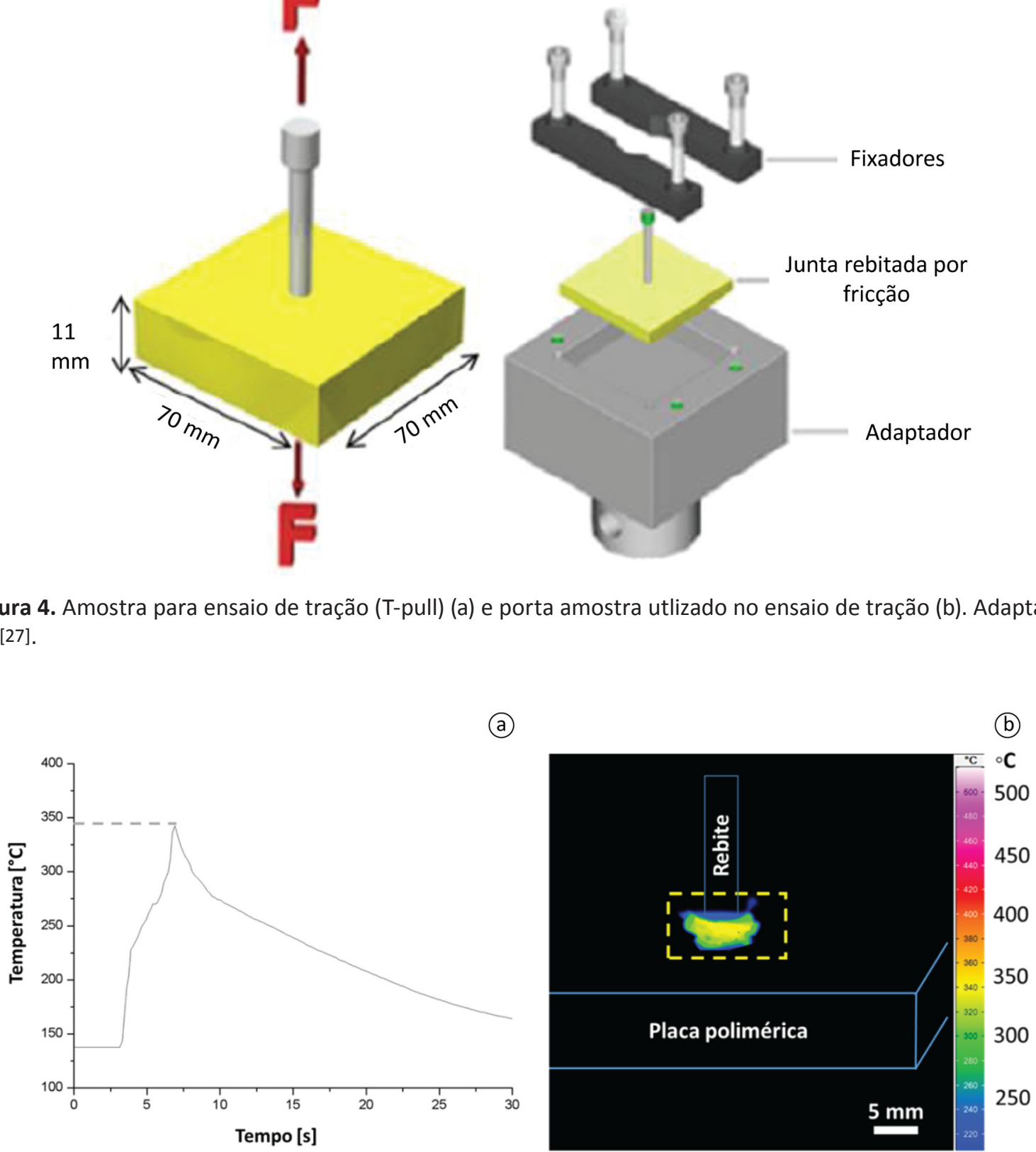

Figura 5. Exemplo da evolução da temperatura durante o processo medido por termografia de infravermelho para a condição C2 (a). Exemplo de um termograma mostrando a temperatura máxima da rebarba do polímero amolecido sendo expelido para fora da placa polimérica para a condição C2. O retângulo amarelo é correspondente à área de medição (b).

A Figura 6 apresenta a temperatura máxima processual atingida para cada condição de rebitagem por friç̧ão empregada (Tabela 1). Nota-se uma grande variação nas temperaturas máximas atingidas durante o processo. A condição C3, que empregou VR de $15000 \mathrm{rev} / \mathrm{min}$, atingiu $375 \pm 5^{\circ} \mathrm{C}$, a condição $\mathrm{C2}$ (VR=12500 rev $/ \mathrm{min}$ ) atingiu $344 \pm 6^{\circ} \mathrm{C}$ e a condição $\mathrm{C} 1$ (VR $\left.=10000 \mathrm{rev} / \mathrm{min}\right), 291 \pm 6^{\circ} \mathrm{C}$. Estudos recentes mostraram que alguns parâmetros do processo exercem influência significativa na geração de calor, sendo que a velocidade de rotação (VR) é o mais influente $[18,28,29]$. 
Rebitagem por Friç̧ão ("FricRiveting”) de Liga de Alumínio 6056 T6 e Poliamida 6: Influência da Velocidade de Rotação na Formação da Zona de Ancoragem e no Desempenho Mecânico

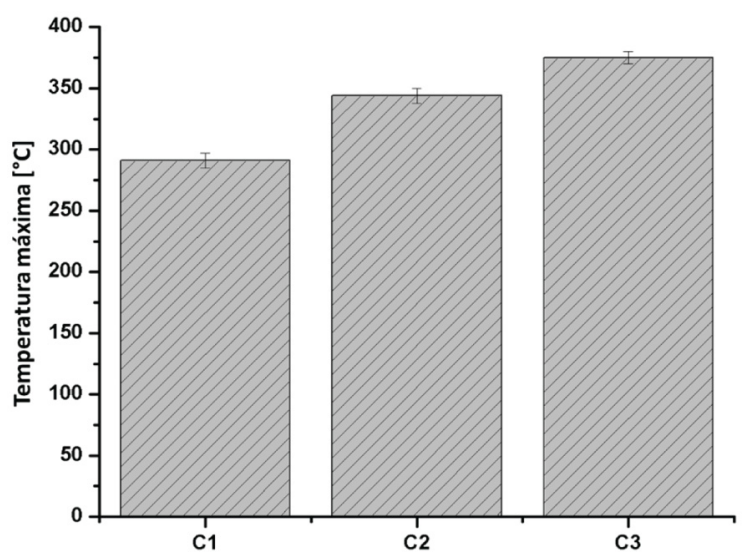

Figura 6. Temperaturas máximas atingida no processo de rebitagem por fricção das juntas de AA 6056-T6 e PA6.

Sabe-se que a evolução da temperatura no processo de rebitagem por fricção é dependente das propriedades térmicas dos materiais de base e do nível de calor friccional gerado em função da combinação dos parâmetros processuais. Essa dependência existe, pois a energia gerada pela fricção dos componentes transforma-se e acumula-se em forma de calor na ponta do rebite metálico, devido à baixa condutividade do polímero. Um modelo matemático (Equação 2) para o calor gerado durante o processo de rebitagem por friç̧ão foi proposto por Amancio [27].

$$
Q=\left[\left(\frac{2}{3} * \mu * P(r)\right)+\frac{\eta * V_{\max }}{H}\right] * V_{\max }
$$

Na Equação 2, $\mu$ é o coeficiente de atrito cinemático, $P(r)$ é a distribuição de pressão normal na ponta do rebite, $\eta$ é a viscosidade do polímero fundido, $V_{\max }$ é a máxima velocidade tangencial do rebite obtida a partir da velocidade angular $(\omega)\left(V_{\max }=\omega^{*} \mathrm{R}\right)$, sendo que $\mathrm{R}$ é o raio inicial do rebite e $\mathrm{H}$ é a largura média da camada polimérica consolidada ao redor do rebite metálico.

A Equação 2 considera que o calor é gerado principalmente pelo cisalhamento no polímero fundido/amolecido, no caso de termoplásticos, ou seja por dissipação viscosa [26]. Nota-se que a velocidade de rotação $\left(\mathrm{V}_{\max }\right)$ tem uma influência quadrática sobre o aquecimento. A partir Equação 2, conclui-se que a condição com maior geração de calor foi C3 (15000 rev/min) e a de menor foi C1 (10000 rev/min) devido à maior velocidade de rotação de C3. Desse modo os valores de temperaturas processuais seguem a tendência esperada e reportada na literatura para outros materiais de base [28,29].

\subsection{Influência da velocidade de rotação na formação da zona de ancoragem}

A deformação plástica da ponta do rebite metálico é diretamente dependente do calor gerado durante o processo de rebitagem por fricção. Em condições de união de maior aporte térmico, a ponta do rebite se deforma plasticamente com maior facilidade. A Figura 7 apresenta as seções transversais típicas das regiões de união das três condições de união estudadas.

Adicionalmente à Figura 7 nota-se na Figura 8 que a alteração da VR de $10000 \mathrm{rev} / \mathrm{min}$ para $12500 \mathrm{rev} / \mathrm{min}$ e $15000 \mathrm{rev} / \mathrm{min}$ gerou aumentos pouco expressivos na profundidade de penetração do rebite (H) $(6,7 \pm 0,2 \mathrm{~mm}$ para $\mathrm{C} 1,7,9 \pm 0,3 \mathrm{~mm}$ para $\mathrm{C} 2$ e 7,8 $\pm 0,3 \mathrm{~mm}$ para a $\mathrm{C} 3$ ) enquanto aumentos significativos na largura (W) da extremidade do rebite foram observados $(4,6 \pm 0,2 \mathrm{~mm}$ para $\mathrm{C} 2,6,4 \pm 0,4 \mathrm{~mm}$ para $\mathrm{C} 2$ e 9,7 $\pm 0,5 \mathrm{~mm}$ para a C3). Em estudos anteriores [18,28] foi observado que um aumento na VR gerava juntas com maior profundidade de inserção do rebite metálico $(\mathrm{H})$. Isso ocorre, pois, com um maior aporte térmico, o termoplástico tem a sua viscosidade reduzida beneficiando assim a penetração do rebite metálico. No entanto, neste estudo foi observado que o aumento da VR não resulta em alteração significativa da penetração do rebite. Por outro lado, a deformação da ponta do rebite (W) aumentou 37\% quando a VR foi aumentada de $10000 \mathrm{rev} / \mathrm{min}$ para $12500 \mathrm{rev} / \mathrm{min}$ e $52 \%$ com o aumento de VR para $15000 \mathrm{rev} / \mathrm{min}$. Neste caso, com maior calor gerado devido ao aumento da VR, além do 

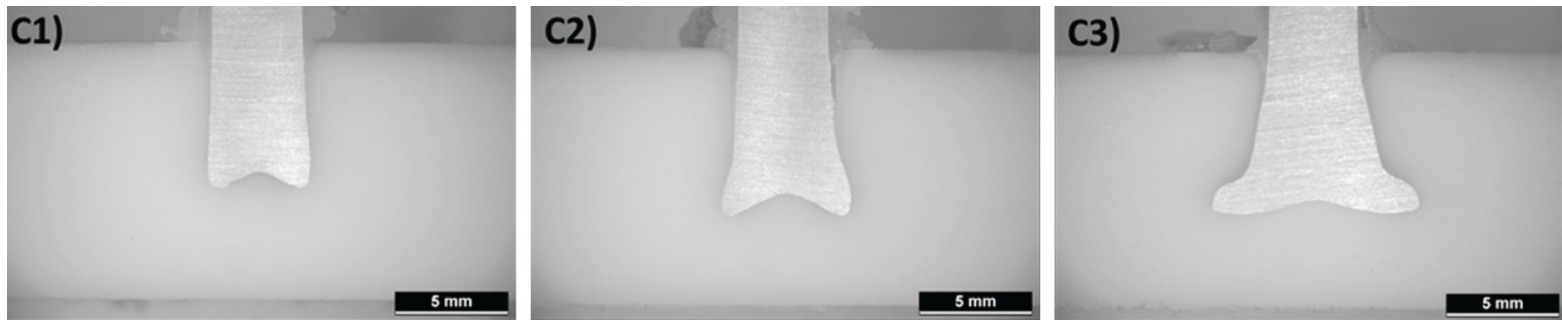

Figura 7. Seções transversais de juntas AA 6056 T6-PA6 produzidas com baixa (10000 rev/min) (C1), média (12500 rev/min) (C2) e alta (15000 rev/min) (C3) velocidade de rotação.

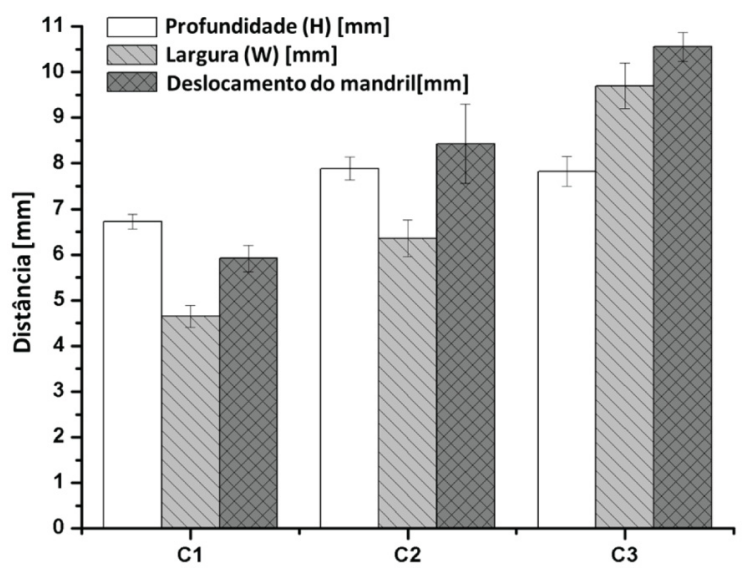

Figura 8. Valores de deslocamento axial do mandril durante a fase de fricção, profundidade (H) e deformação (W) do rebite metálico inserido na placa polimérica e para as condições analisadas.

termoplástico reduzir sua viscosidade a ponta do rebite também se deforma plasticamente com maior facilidade. Assim, para as juntas AA 6056 T6-PA6, é provável que a deformação plástica da ponta do rebite metálico para a condição de maior aporte térmico (C3) ocorra já durante a fase de fricção.

O equipamento de rebitagem usado possui sensor de deslocamento axial do mandril, com isso é possível comparar a profundidade de penetração $(\mathrm{H})$ do rebite metálico das juntas (medidos diretamente das secções transversais das juntas) com o deslocamento axial do mandril durante a fase de friç̧ão. A profundidade de penetração $(H)$ é alcançada após as duas fases do processo, a de fricção e de forjamento. Teoricamente, durante a fase de friç̧ão, espera-se a ausência de deformação plástica na ponta do rebite; logo, a profundidade de penetração $(\mathrm{H})$ deve ser maior ou igual ao deslocamento do mandril durante a fase de fricção. A Figura 8 apresenta os dados do deslocamento do mandril até o final da fase de fricção $(3500 \mathrm{~ms})$ e da profundidade de inserção do rebite metálico nas juntas AA 6056 T6-PA6 para todas as condições estudadas. Nota-se que na condição com menor aporte térmico (C1) o deslocamento final do mandril na fase de friç̧ão $(5,9 \pm 0,3 \mathrm{~mm})$ é menor do que a profundidade do rebite metálico nas juntas $(6,7 \pm 0,2 \mathrm{~mm})$. Para a condição com aporte térmico médio (C2) o deslocamento do mandril $(8,4 \pm 0,7 \mathrm{~mm})$ é um pouco maior do que a profundidade de inserção do rebite $(7,9 \pm 0,3 \mathrm{~mm})$, evidenciando um possível início de deformação plástica do mesmo durante a fase friç̧ão. Já para a condição com maior aporte térmico (C3) o deslocamento do mandril é visivelmente maior $(10,5 \pm 0,4 \mathrm{~mm})$ que a profundidade de inserção do rebite $(7,8 \pm 0,3 \mathrm{~mm})$ indicando o início da deformação plástica da ponta do rebite durante a fase friç̧ão. Adicionalmente, esse processo de deformação pode ser evidenciado ao se comparar as condições $\mathrm{C} 2$ e C3 onde as profundidades $(\mathrm{H})$ são estatisticamente iguais $(7,9 \pm 0,3 \mathrm{~mm}$ para $\mathrm{C2}$ e 7,8 $\pm 0,3 \mathrm{~mm}$ para a C3) mas o deslocamento do mandril é bem maior para a condição C3 $(8,4 \pm 0,7 \mathrm{~mm}$ para C2 e 10,5 $\pm 0,4 \mathrm{~mm}$ para C3). Assim, têm-se provavelmente a ocorrência de deformação plástica durante a fase de fricção devido ao maior aporte térmico, fato esse reportado pela primeira vez na literatura. Tal argumento foi confirmado através de experimentos auxiliares de "stop-action" (parada do processo em diversas etapas para análises microestruturais instantâneas da junta) realizados em estudos complementares a serem publicados separadamente. 


\subsection{Influência da velocidade de rotação no desempenho mecânico das juntas rebitadas por fricção}

A Figura 9 apresenta os valores de força máxima de tração das juntas produzidas nas condições C1, C2 e C3 e do rebite metálico. Comparando as condições C1-C3 observa-se que, em termos de resistência mecânica, a VR tem uma influência significativa. Isso ocorre, pois, uma junta gerada com maior aporte térmico possui uma maior deformação plástica da ponta do rebite metálico, melhorando assim sua ancoragem, como ilustrado na Figura 7.

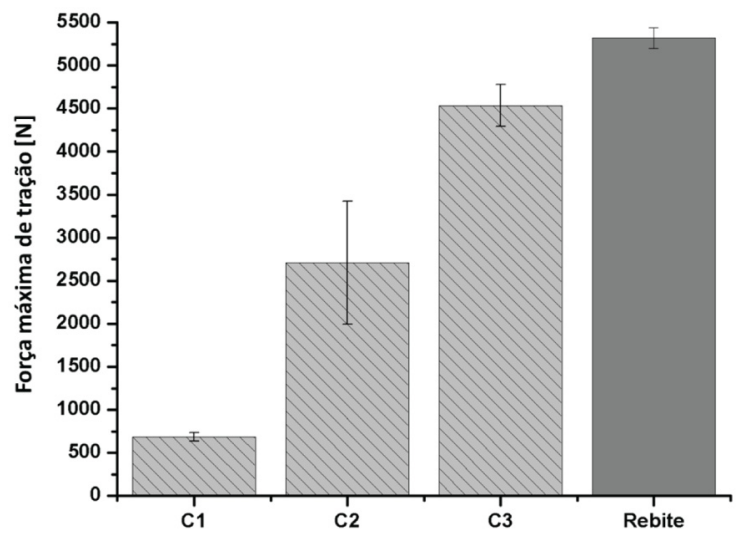

Figura 9. Força máxima de tração das juntas AA 6056 T6-PA6 e razão volumétrica de ancoramento para as condições analisadas.

A razão volumétrica de ancoragem estima quantitativamente a ancoragem do rebite metálico na placa polimérica. Uma junta com alto valor de razão volumétrica de ancoragem terá uma maior resistência à tração, conforme relatado por Blaga et al. [15] para juntas Ti gr.2-PEI FV e por Rodrigues et al. [17] para juntas AA 2024 T351-PC rebitadas por fricção. A Figura 10 mostra a relação entre a razão volumétrica de ancoragem e a força máxima de tração das juntas AA 6056 T6-PA6. Como evidenciado, a relação entre a força máxima de tração e a razão volumétrica de ancoragem é linear, seguindo a Equação 3. A confiabilidade do modelo foi avaliada a partir dos valores do coeficiente de aproximação da regressão $\left(R^{2}\right)$. Neste caso, $R^{2}$ é igual a 0,99 o que indica uma boa concordância do modelo com os dados experimentais. Além disso, nota-se que altos valores de razão volumétrica resultaram em juntas com elevada resistência mecânica, seguindo a tendência reportada na literatura $[15,17]$.

Força máxima de tração $[\mathrm{N}]=7152 * \mathrm{RV}-767$

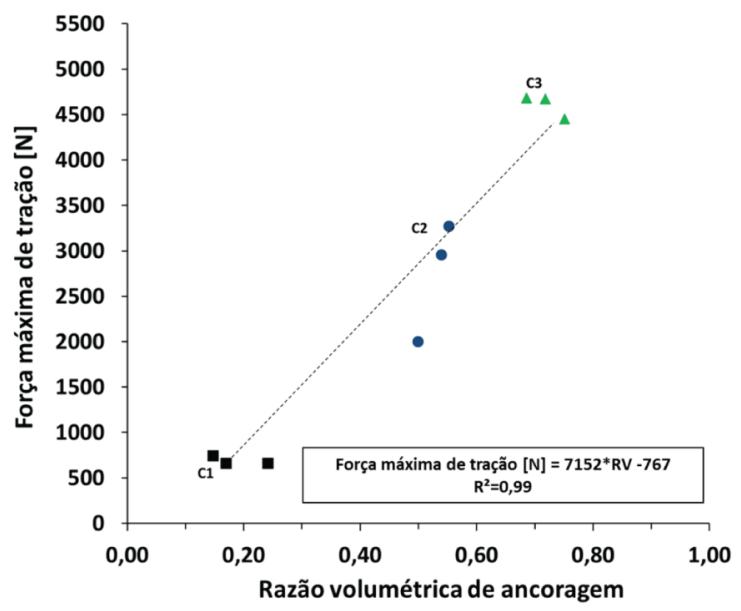

Figura 10. Relação entre força máxima de tração das juntas AA 6056 T6-PA6 e razão volumétrica de ancoragem (RV). 
A Figura 11 apresenta os dois tipos de fraturas observados para as juntas com as condições estudadas. Nas condições $\mathrm{C} 1$ e C2 o rebite metálico foi arrancado da placa polimérica (full-rivet pull-out), atingindo somente $13 \pm 1 \%$ da força máxima de tração do rebite metálico para a condição C1 e $51 \pm 13 \%$ para a C2 (Figura 11a), já que a falha final não ocorreu no rebite. A junta produzida na condição C3 apresentou fratura dúctil do rebite metálico fora da placa polimérica (through the rivet) (Figura 11b) atingindo $85 \pm 5 \%$ da força máxima de tração do rebite metálico. A fratura no rebite metálico na condição C3 ocorreu com uma força menor em comparação ao material de base. Isso se deve a fenômenos de recozimento no rebite metálico, como recuperação e recristalização dinâmica na zona plasticamente deformada, os quais estão relacionados com as temperaturas e taxas de deformação atingidas durante o processo, tal como reportado anteriormente por Amancio et al. [10] e Rodrigues et al. [17] . Já na área não deformada plasticamente, porém afetada termicamente pelo processo, estudos evidenciaram uma redução no valor de microdureza Vickers devido à uma possível recuperação estática, solubilização e/ou a coalescência de precipitados [10,17]. Juntas de AA 2024 T351-PEl atingiram 93\% da força máxima de tração do rebite metálico [26] as juntas de AA 2024 T351-PC atingiram 89\% da força máxima de tração do rebite metálico [17]. Estas transformações no rebite metálico estão atualmente sob investigação e não são o foco do presente trabalho.
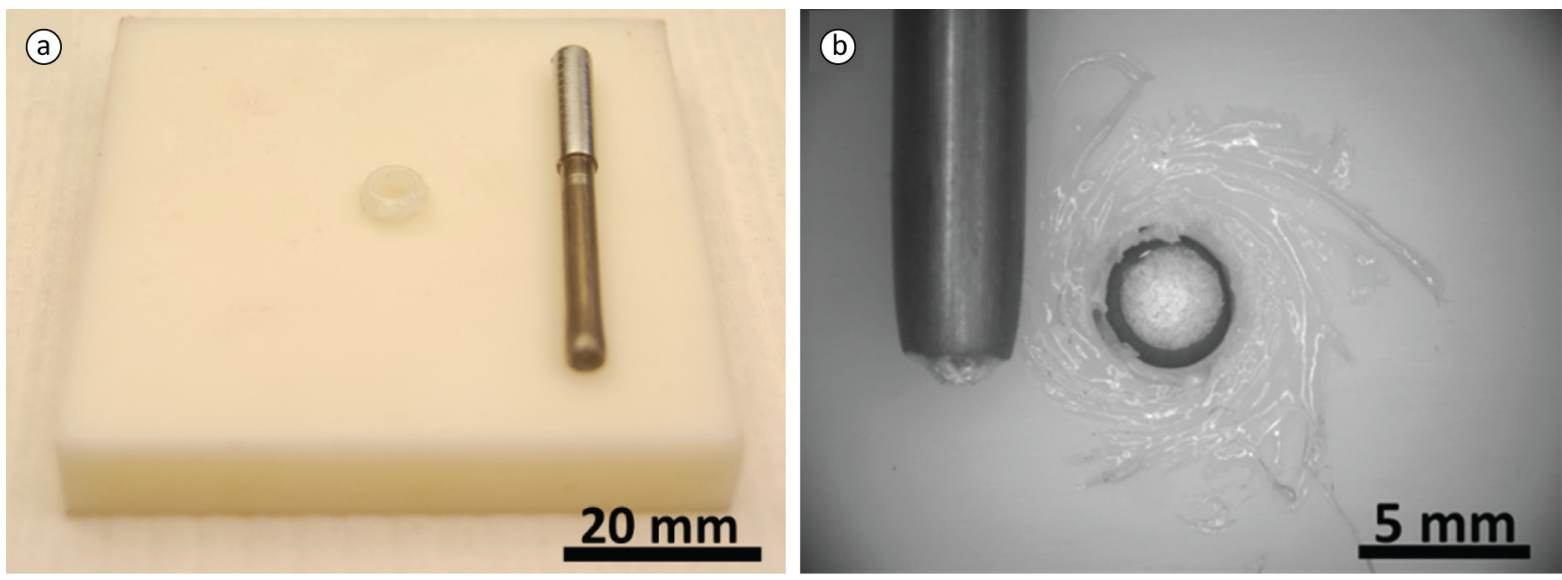

Figura 11. Fratura na qual o rebite é arrancado da placa polimérica (full-rivet pull-out) (a) e fratura dúctil do rebite metálico fora da placa polimérica (through the rivet) caracterizada pela forma taça e cone da superfície de fratura (b).

\section{Conclusões}

A viabilidade técnica da Rebitagem por Friç̧ão para a união de rebite de liga de alumínio 6056-T6 e placa de poliamida 6 (PA6) foi comprovada. Além disso, neste estudo, a influência da velocidade de rotação (VR) do rebite na temperatura máxima processual e no desempenho mecânico das juntas foi analisada.

Juntas produzidas com a maior velocidade de rotação (15000 rev/min) apresentaram maior deformação plástica da ponta do rebite metálico. Isso está associado à maior energia gerada pela fricção dos componentes que se transforma e se acumula em forma de calor na ponta do rebite metálico devido à baixa condutividade do polímero.

Notou-se pela primeira vez evidências de que a deformação plástica do rebite metálico está ocorrendo durante a fase de fricção em condições de alto aporte térmico. Isso é importante para o entendimento da física do processo que anteriormente abordava a formação da zona de ancoragem somente durante a fase forjamento. Tal fato pode encurtar o ciclo processual reduzindo ou eliminando a fase de forjamento.

A maior plastificação da ponta do rebite metálico gerou melhor ancoragem do rebite com valores de razão volumétrica de ancoragem de 0,71 $\pm 0,02$. As juntas AA 6056 T6-PA6 seguiram a tendência mostrada na literatura, na qual a resistência à tração é diretamente proporcional à razão volumétrica de ancoragem.

Portanto, verificou-se que a velocidade de rotação influencia diretamente na resistência à tração das juntas rebitadas por fricção, onde um aumento de $50 \%$ na velocidade de rotação (5000 rev/min) aumentou a força máxima de tração das juntas de $685 \pm 48 \mathrm{~N}$ para $4536 \pm 246 \mathrm{~N}$. 
Rebitagem por Fricção ("FricRiveting") de Liga de Alumínio 6056 T6 e Poliamida 6: Influência da Velocidade de Rotação na Formação da Zona de Ancoragem e no Desempenho Mecânico

\section{Agradecimentos}

Os autores agradecem o suporte financeiro e técnico da Helmholtz Association da Alemanha através do Young Investigator Group “Advanced Polymer-Metal Hybrid Structures” (Helmholtz Association - Grant No. VH-NG-626), ao material fornecido pela EJOT GmbH, Alemanha, e ao CNPq pela bolsa de produtividade em pesquisa concedida ao Prof. Dr. Leonardo B. Canto (CNPq - Processo 304169/2014-5).

\section{Referências}

[1] DuPont. Innovative ideas for metal replacement. Wilmington: DuPont; 2015 [acesso em 30 abr. 2015]. Disponível em: http:// www.dupont.com/products-and-services/plastics-polymersresins/articles/innovative-ideas-for-metal-replacement.html

[2] Amancio-Filho ST, Santos JF. Joining of polymers and polymermetal hybrid structures: recent developments and trends. Polymer Engineering and Science. 2009;49(8):1461-1476. http:// dx.doi.org/10.1002/pen.21424.

[3] Kah P, Suoranta R, Martikainen J, Magnus C. Techniques for joining dissimilar materials: metals and polymers. Reviews on Advanced Materials Science. 2014;36(2014):152-164.

[4] Niu M. Airframe structural design: practical design information and data on aircraft structures. California: Conmilit Press; 1988.

[5] Higgins A. Adhesive bonding of aircraft structures. International Journal of Adhesion and Adhesives. 2000;20(5):367-376. http:// dx.doi.org/10.1016/S0143-7496(00)00006-3.

[6] Wegman F, Twisk JV. Surface preparation techniques for adhesive bonding. Waltham: Elsevier Inc; 2012.

[7] Baldan A. Adhesively-bonded joints in metallic alloys, polymers and composite materials: mechanical and environmental durability performance. Journal of Materials Science. 2004;39(15):47294797. http://dx.doi.org/10.1023/B:JMSC.0000035317.87118. $\mathrm{ab}$.

[8] Michalos G, Makris S, Papakostas N, Mourtzis D, Chryssolouris G. Automotive assembly technologies review: challenges and outlook for a flexible and adaptive approach. CIRP Journal of Manufacturing Science and Technology. 2010;2(2):81-91. http:// dx.doi.org/10.1016/j.cirpj.2009.12.001.

[9] Camanho FL. Stress analysis and strength prediction of mechanically fastened joints in FRP: a review. Composites Part A: Applied Science and Manufacturing. 1997:28(6):529-547.

[10] Amancio-Filho ST. Rebitagem por fricção ('FricRiveting'). Desenvolvimento de uma nova técnica de união para juntas híbridas do tipo polímero-metal. Parte I: processo e microestrutura. Soldagem \& Inspeção. 2011;16(4):387-395. http://dx.doi.org/10.1590/S0104-92242011000400010.

[11] Goushegir SM, Santos JF, Amancio-Filho ST. Friction Spot Joining of aluminum AA2024/carbon-fiber reinforced poly(phenylene sulfide) composite single lap joints: microstructure and mechanical performance. Materials \& Design. 2014;54:196-206. http:// dx.doi.org/10.1016/j.matdes.2013.08.034.

[12] Abibe AB, Amancio-Filho ST, Santos JF, Hage E Jr. Mechanical and failure behaviour of hybrid polymer-metal staked joints. Materials \& Design. 2013;46:338-347. http://dx.doi.org/10.1016/j. matdes.2012.10.043.

[13] Abibe AB, Santos JF, Amancio-Filho ST. Friction staking: a noval staking joining method for hybrid structures. In: Society of Plastics Engineers. Proceedings of the Technical Conference \& Exhibition - ANTEC 2014; 2014 Abr 28-30; Las Vegas, USA. Newtown: Society of Plastics Engineers; 2014. p. 1755-1761.

[14] Amancio TS Fo, Beyer M, Santos JF. inventores; GkssForschungszentrum Geesthacht Gmbh, cessionário. Method of connecting a metallic bolt to a plastic workpiece. United States patent US 7575149 B2. 2009 Ago 18.

[15] Blaga L, Bancilă R, Santos JF, Amancio-Filho ST. Friction Riveting of glass-fibre-reinforced polyetherimide composite and titanium grade 2 hybrid joints. Materials \& Design. 2013;50:825-829. http://dx.doi.org/10.1016/j.matdes.2013.03.061.

[16] Proenca B, Blaga LA, Santos JF, Canto LB, Amancio-Filho ST. Force controlled friction riveting of glass fiber reinforced polyamide 6 and aluminum alloy 6056 hybrid joints. In: Society of Plastics Engineers. Proceedings of the Technical Conference Exhibition ANTEC 2015; 2015 Mar 23-25; Orlando, USA: Society of Plastics Engineers; 2015.

[17] Rodrigues CF, Blaga LA, Santos JF, Canto LB, Hage E Jr, Amancio-Filho ST. FricRiveting of aluminum 2024-T351 and polycarbonate: temperature evolution, microstructure and mechanical performance. Journal of Materials Processing Technology. 2014;214(10):2029-2039. http://dx.doi.org/10.1016/j. jmatprotec.2013.12.018.

[18] Altmeyer J, Santos JF, Amancio-Filho ST. Effect of friction riveting process parameters on the joint formation and performance of ti alloy/ short-fibre reinforced polyether ether ketone joints. Materials \& Design. 2014;60:164-176. http://dx.doi.org/10.1016/j. matdes.2014.03.042.

[19] Borba NZ, Blaga LA, Santos JF, Canto LB, Amancio-Filho ST. Friction riveting of pultruded thermoset glass fiber reinforced polyester composite and Ti6Al4V hybrid joints. In: Society of Plastics Engineers. Proceedings of the Technical Conference \& Exhibition - ANTEC 2014; 2014 Abr 28-30; Las Vegas, USA. Newtown: Society of Plastics Engineers; 2014.

[20] Maxwell J. Plastics in the automotive industry. Cambridge: Woodhead Publishing Ltd; 1994.

[21] Iwayama I, Kuwabara T, Nakai Y, Takaki Y, Kitamura S-I, Saito H. 6056 aluminum alloy wire for automotive fasteners. SEI Technical Review. 2013;77:74-78.

[22] Meng C. Effect of preheating condition on strength of AA6060 Aluminum Alloy for extrusion [tese de doutorado]. Nova Zelândia: Auckland University of Technology; 2010.

[23] Marco H. Polyamides, polyesters and elastomeric thermoplastics: a sustainable perspective in modern industry. Polímeros. 2012;22(2):107-110.

[24] Zimmerman MIK, Kohan MI. Nylon-selected topics. Journal of Polymer Science Part A: Polymer Chemistry. 2001;39(15):25652570. http://dx.doi.org/10.1002/pola.1233.

[25] Associação Brasileira de Normas Técnicas. NBR ISO 6892. Materiais metálicos - ensaio de tração à temperatura ambiente. Rio de Janeiro: ABNT; 2002.

[26] Amancio-Filho ST. Rebitagem por fricção ('FricRiveting'). Desenvolvimento de uma nova técnica de união para juntas híbridas do tipo polímero metal. parte ii: propriedades térmicas e mecânicas. Soldagem \& Inspeção. 2011;16(4):396-404. http:// dx.doi.org/10.1590/S0104-92242011000400011.

[27] Amancio-Filho ST. Friction riveting: development and analysis of a new joining technique for polymer-metal multi-materials structures [tese de doutorado]. Geesthacht: Technischen 
Universitäat Hamburg-Harburg, GKSS-Forschungszentrum Geesthacht; 2007.

[28] Rodrigues C. Rebitagem por fricção de alumínio 2024-T351 em policarbonato [dissertação de mestrado]. São Carlos: Universidade Federal de São Carlos; 2014.
[29] Amancio-Filho ST, Roeder J, Nunes SP, Santos JF, Beckmann F. Thermal degradation of polyetherimide joined by friction riveting (FricRiveting). Part I: influence of rotation speed. Polymer Degradation \& Stability. 2008;93(8):1529-1538. http:// dx.doi.org/10.1016/j.polymdegradstab.2008.05.019. 